\title{
Design and implementation of the subsystem subject to emission of multicomponent high-frequency signals to ensure its reliability
}

\author{
Milad Daneshvar ${ }^{1}$, Naser Parhizgar ${ }^{2}$, Homayoon Oraizi $^{3}$ \\ ${ }^{1,2}$ Department of Electrical Engineering, Shiraz Branch, Islamic Azad University, Shiraz, Iran \\ ${ }^{3}$ Department of Electrical and Computer Engineering, Iran University of Science and Technology, Tehran, Iran
}

\begin{tabular}{|c|c|}
\hline Article Info & ABSTRACT \\
\hline Article history: & \multirow{11}{*}{$\begin{array}{l}\text { Telecommunication systems, especially digital ones, are mostly known to be } \\
\text { immune to noise given their extensive range of applications. This study } \\
\text { aimed to investigate the methods and tools used for the analysis of } \\
\text { multicomponent signals input to high-frequency digital subsystems, } \\
\text { including the analysis of changes in its electrical behavior. This research } \\
\text { mainly focuses on analyzing a high-frequency telecommunication subsystem, } \\
\text { recording the results, investigating the system behavior against signals with } \\
\text { different amplitudes and phases, detecting the received signals, and } \\
\text { measuring the phase differences. The study extended the mono-component } \\
\text { signals to multi-component signals and accurately extracted the statistical } \\
\text { signal specifications using analytic signals in the time-frequency domain. } \\
\text { To this end, a method was proposed based on the switch matrix to relate the } \\
\text { different components and parameters, and also a mathematical model based } \\
\text { on the state-space equations was employed to evaluate the nonlinear system } \\
\text { modes. Given that the decoupling of measurement parameters is a problem to } \\
\text { be tackled from multiple aspects, the costs and test durations were also taken } \\
\text { into calculations in addition to considering all the detection methods for } \\
\text { interference signals, reliability and time under test. }\end{array}$} \\
\hline Received Jan 6, 2019 & \\
\hline Revised Apr 19, 2019 & \\
\hline Accepted Apr 30, 2019 & \\
\hline & \\
\hline Keywords: & \\
\hline High-frequency signals & \\
\hline Mono-component & \\
\hline Multicomponent & \\
\hline Reliability & \\
\hline Subsystems & \\
\hline
\end{tabular}

Copyright $@ 2019$ Institute of Advanced Engineering and Science. All rights reserved.

\section{Corresponding Author:}

Naser Parhizgar,

Department of Electrical Engineering,

Shiraz Branch, Islamic Azad University,

Shiraz, Iran

Email: naserpar@yahoo.com

\section{INTRODUCTION}

The design of high-frequency systems is considered an important task is given the recent advancements in their analysis. In the past, systems operated at frequencies no higher than a few megahertz $(\mathrm{MHz})$, and their efficiency was not affected by the configuration of elements and tracks and their interconnections. However, today, new phenomena are likely to occur considering the increase in system frequencies up to a few gigahertz $(\mathrm{GHz})$, such that inappropriate system design and lack of knowledge on the respective principles in this regard can easily cause interference in the system performance. The present study aimed at finding signals affecting the subsystem at different angles. The power of the received signals is not the subject of discussion, because, in that case, the density of different signals and the intensity of each signal in different situations must be measured. The most destructive type of signals is the RF signal radiated on the subsystem surface. It should be noted that measuring such signals is always accompanied by ambiguities due to its unknown nature and randomness. Signal processing allows for the decomposition of a signal to its different components by proper weighting to simulate each component with the desired distribution. To prevent an increase in the computational iterations, which consequently increases the simulation time in 
the analysis of signal components, the number of meshes used in the subsystem was reduced by separating the components. As a matter of fact, the components separation of the signal is expressing an important task in multi-component signal applications. In the initial state without any hypothesis, the signal is analytic and mono-component. In signal Analytic Methods, we use mono-component signals to demonstrate nonstationary and nonlinear signals rather than the Fourier transform solutions, such as functions used in empirical method decomposition. Multi-component signal analysis is needed explicitly to analysis the systems affected by electromagnetic interference. In the case of such signals, instead of a single signal with a specific amplitude and phase, multiple signals with random amplitudes and phases enter the system and affect its behavior. In practice, in most studies and simulations available in the literature, the analysis is conducted for a single signal, and the results are then extended to the actual calculations [1-3]. The analysis of the mono-component empirical signal is proposed in the literature to solve interference problems. However, the complexity of signal estimation will be increased. Obviously, the definition of the monocomponent signal provides a partial portion of information. However, we need all the information and for that matter use multi-component signals. If we have a mono-component signal, it provides a method for quantitative definitions and characterizations.

Whenever the components are extracted, quantitative measurements keep the components in order, which is considered in the simulation of component decomposition method. The proposed idea creates an important phase due to multi-component signals. An objective of this paper is to provide a method for measuring components by implementing a decomposition algorithm. Here various methods about component separation are described, such as empirical analysis decomposition and local decomposition algorithm. For the analysis of mono-component signal by a decomposition algorithm, the interference is achieved through multi-component signals [4-7].

The other methods are based on Gabor filtering and energy separation, but the interference will be embedded in the separated components. The technique separates a multi-component signal as non-stationary into narrow-band components. The general premise in the past was that the Fourier transform is inefficient for the non-stationary and nonlinear analysis, which is right in most papers. An adaptive technique may be used in a mono-component signal application according to the fundamental solution analysis to include its effects for nonlinear and non-stationary time sequences. The Fourier separation method is a particular type of mono-component signal separation. It many papers an adaptive Fourier separation method is presented based on an energy criterion. Adaptive Fourier separation method and its variations lead to fast separation of functions into mono-components, which can extract a solution to the formulation problem of an optimal estimation [8]. The methods used in the time-frequency domain are expressed by two viewpoints: parametric and non-parametric time-frequency analysis; the first form has signal-dependent parameters and the second form is defined without signal-dependent parameters. Parameterized methods consider an additional parameterized signal with the frequency modulated information. A noisy multi-component signal can be shown as the sum of noise and sinusoidal functions, which is defined as [9],

$$
S(t)=\sum_{l=1}^{N} S_{l}(t)+n_{w}(t)
$$

where $\mathrm{n}_{\mathrm{w}}(\mathrm{t})$ is the Gaussian noise with mean $=0$, variance $=\sigma 2$ and $\mathrm{N}$ is the number of components. In the analytic the signal form, $S_{1}(t)$ is the L-th component of the signal, $S_{1}(t) \in L_{2}(R)$ and can be presented through the Hilbert transform theory:

$$
Z(t)=\sum_{l=1}^{N} Y_{l}(t) e^{j \beta_{l}(t)}+Z_{n}(t)
$$

where every instantaneous frequency is $\mathrm{f}_{l}=\left(\beta_{1}^{\prime}(\mathrm{t})\right) / 2 \pi$. As $\mathrm{Y}_{1}(\mathrm{t})$ is variable and varies with the instantaneous frequency and is considered to have a constant amplitude. We can use the properties of (1), where $\mathrm{N}=1$, then the signal reproduces to a mono-component signal, thus $S(t)=S_{1}(t)+n_{w}(t)$. The model of signal applies to noisy multi-component signals [10]. Between the signal decomposition methods, the Hilbert transform plays empirical mode decomposition to present the multi-component signal with a group of functions. The Hilbert transform is used to convert the time domain signal into an analytic signal. Signals are often vectors of complex numbers. The vector of a simple sine wave in the time domain consists of complex numbers, of which the phase is changing in time series concerning the main sine wave with a constant magnitude. This method is applied to get the instantaneous phase lag between two signals. Its main limitation is that the input signal needs to be mono-component [11]. Therefore, you may have to filter out any interference. It is estimated for mono-component signals only; however, it is extensible for multi-component signals using

Design and implementation of the subsystem subject to emission of multicomponent ... (Milad Daneshvar) 
changes in signal concepts. The specification of mono-component signals includes single sine waves using the fast Fourier transform. For the estimation of frequencies as functions of time, we can use spectrogram [12]. In several papers, it is referred to The Hilbert-Huang transform that is an empirical approach and has been tested and validated. In almost all the cases studied, the Hilbert-Huang transform gives results much sharper than any of the theoretical analysis methods in a time-frequency-energy demonstration. In [13], to define phase derivatives of analytic signals and a new type of partial derivatives for phase computation (called Hardy-Sobolev derivatives) for input signals in the related Sobolev spaces. Based on the study of several types of phase derivatives and their characterization, [13] generally works on the three subjects:

- Extend the state between the instantaneous frequency and the Fourier transform of frequency response and the related ones for input signals to those in the Sobolev spaces.

- Based on the phase derivative theory and the recent results of phase derivatives, the theoretical structure of the minimum phase and all-pass filters are established. Both the continuous and discrete signals types are considered.

- To study a partial type of time-frequency function suitable for mono-component, called transient timefrequency distribution. For multi-components, we need some supplementary study [14].

The present study attempted to develop a method for simultaneous assessment of multiple signals so that to increase the efficiency through multi-component signals and using signal modeling. New components may be added to the signal when exposed to any interference. The mono-component signal analysis is not an option and the currently common methods, such as analytic signal modeling, should be used for the envelope, phase, and instantaneous frequency calculations. Moreover, envelope detectors and implementations of phase detectors were used to determine the unknown signal parameters and separate and determine the signal phases are entering the system $[15,16]$. We study multi-component signals for analytic signals modeling. Unlike the Sin, Cos, and single-pulse functions, which are bounded in one domain and unbounded in another (from a duration perspective), the Gaussian function is localized in both time and frequency domains.

Additionally, due to its infinite differentiability, the Gaussian function and its Fourier transform were used in the calculations given their more straightforward analytical applications compared to other purposes. In spectral computations, $|\mathrm{S}(\mathrm{w})|^{2}$ denotes the power spectrum, which defines the signal power at different frequencies and is extensively employed in analytic signal calculations. In other words, $|\mathrm{S}(\mathrm{w})|^{2}$ represents the distribution of signal energy in the frequency domain. For secure computation in the frequency domain, we use the Fourier transform. Detecting signals that affect different subsystems at different angles is of special importance in the design of high-frequency systems, which is why signal detection has been extensively discussed in the literature. Signal detection is a data analysis method for decision-making problems involving uncertainty (noise) $[2,6,12]$. The switch matrix containing all information about the signal was used in this study to present a new solution. We employed this matrix with a proper weight percentage in $\mathrm{n}$ tests to analyze different scenarios.

\section{SIMULATION OF SIGNAL}

Given that the Fourier transform is an improper integral and based on the Wiener-Khintchine theorem, the signal power is obtained using the autocorrelation function:

$$
|S(w)|^{2}=\int_{-\infty}^{+\infty} r_{s}(\varsigma) e^{-j w \varsigma} d \varsigma
$$

where $r_{s}(\zeta)$ is the signal autocorrelation function. Simulation of the signals input to the subsystem in the time and frequency domains are demonstrated in Figures 1, 2, and 3.

From a mathematical perspective, a unique signal can be expanded in an infinite number of ways, which is among the ambiguities faced in the field of signal analysis. The signals check-in time domain and use results in the frequency domain [16].

$$
s(t)=y(t) \cos (P(t))
$$

where $\mathrm{y}(\mathrm{t})$ is the amplitude modulation, and $\mathrm{P}(\mathrm{t})$ is phase modulation. The existing signals in nature are modeled using (4), meaning that their amplitudes and phases change with time. However, nature does separately provide us with the signal amplitude $y(t)$ and phase $P(t)$. Instead, the left-hand side of the equation is known, which leaves us with the question of how to determine $y(t)$ and $\varphi(t)$ such that the considered signal forms the real part of the complex signal, or in other words, how to determine the complex part such that 
$\mathrm{S}_{\mathrm{r}}+\mathrm{j} \mathrm{S}_{\mathrm{i}}$ are combined( the analytic signals analysis case). As one of the solutions, the Hilbert method can be incorporated with the complex form of the signal [17].

$$
\begin{aligned}
& S(t)=Y(t) \cdot e^{j \varphi(t)}=S_{r}+j S_{i} \\
& S_{r}=Y(t) \cos (\varphi(t))
\end{aligned}
$$

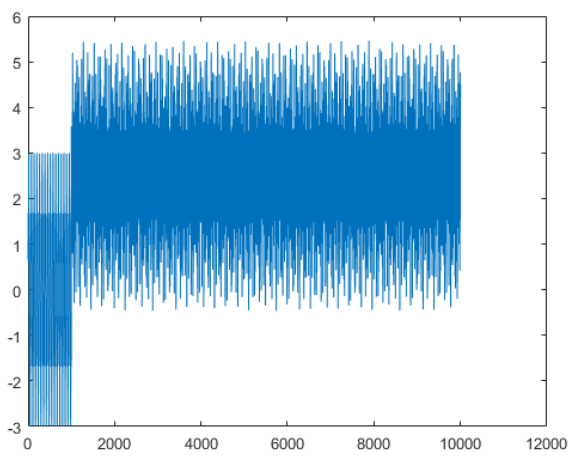

Figure 1. Random signal $\mathrm{X}$ in time domain

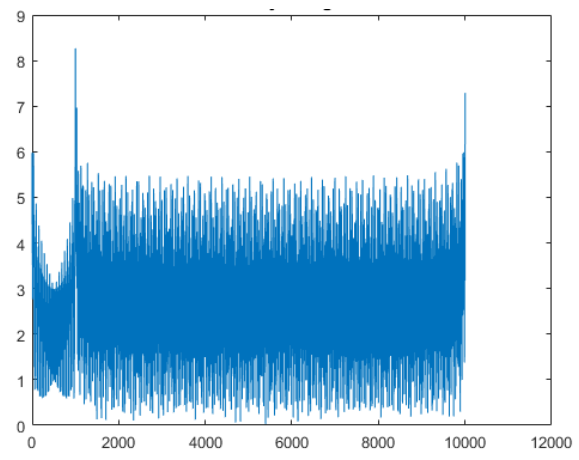

Figure 2. Analytical signal $\mathrm{X}$ in time domain

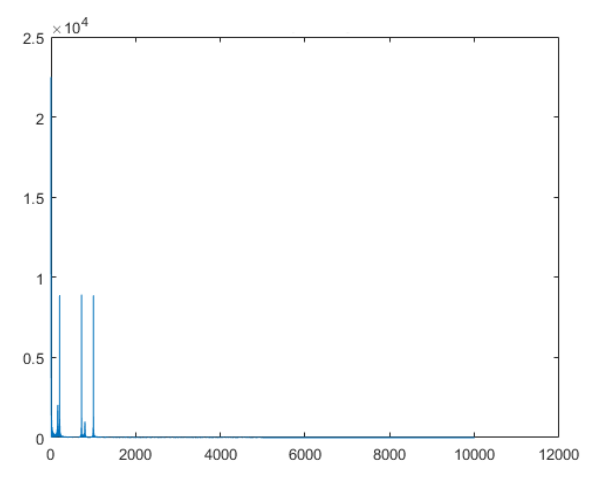

Figure 3. Fast Fourier transform of the analytical signal $\mathrm{X}$ in the frequency range

Although the real part of the complex signal is our considered signal $(y(t) \cos (\mathrm{P}(\mathrm{t})))$, the real amplitude and phase are not necessarily the ones in this real part, which is since the solution to the problem is not unique. By normalizing the signal energy (i.e. $E_{s}=\int_{-\infty}^{+\infty}|s(t)|^{2} d t=1$ ), the term $|\mathrm{s}(\mathrm{t})|^{2}$ can be considered as a probability density function (pdf), through which the statistical specifications of the signal including mean, variance, and higher-order moments can be extracted.

$$
\text { Mean }:<t>=M=\int|s(t)|^{2} d t
$$

where $\mathrm{M}$ represents the center of gravity of the energy in a time domain. The expansion is calculated using variance:

$$
\sigma_{t}^{2}=<t^{2}>-M^{2}
$$

Analysis of signals in the frequency domain due to Fourier Transform application is considerably more comfortable compared to time domain $[18,19]$. Corresponding to the time domain model presented earlier $\left(s(t)=Y(t) e^{j \varphi(t)}\right)$, the frequency model for the signal is expressed as follows:

$$
S(w)=C(w) e^{j \psi(w)}
$$


where $\mathrm{C}(\mathrm{w})$ is spectral amplitude, and $\psi(\mathrm{w})$ is the spectral phase. A signal normalized in the time domain is also normalized in the frequency domain. Hence, we may calculate the mean frequency $\langle w\rangle$ and the variance $\left(\sigma_{w}^{2}\right)$ in the frequency domain. Note that in the frequency domain, variance represents the bandwidth.

$$
\begin{aligned}
& <w>=Q=\int w|s(w)|^{2} d w \\
& \sigma_{w}^{2}=<w^{2}>-Q^{2}
\end{aligned}
$$

We may not be able to calculate the Fourier transform for all signals and extract their statistical specifications. This means that $\mathrm{S}(\mathrm{t})$ is initially required to determine $\mathrm{Q}$ and $\sigma_{w}^{2}$ or the variance of the frequency domain. The Fourier transform does not apply to all signals; we defined our objective to extract the statistical specifications from the time signals and signal components. $\mathrm{s}_{\mathrm{r}}$ denotes a real known signal, and we aim to determine $\mathrm{s}_{\mathrm{i}}$.

$$
\begin{aligned}
& Z(t)=s_{r}+j s_{i}=Y(t) e^{j \varphi(t)} \\
& \varphi^{\prime}(t)=w_{i}(t)=\frac{\left(s_{i}^{\prime} s_{r}-s_{r}^{\prime} s_{i}\right)}{Y^{2}}
\end{aligned}
$$

where $\varphi^{\prime}(\mathrm{t})$ is instantaneous frequency, the imaginary part $\left(\mathrm{s}_{\mathrm{i}}\right)$ can be determined with Analytic method. In this method, an analytic form is considered for the signal. The method is based on this process the spectral frequency of complex signals becomes either zero or the same as the real signal spectrum for positive and negative frequencies, respectively. In the simulation for spectrum power analysis, we use the analytic method [20, 21]. In Figure 4 shows the comprision of power spectral density simulation for two original and Hilbert types.

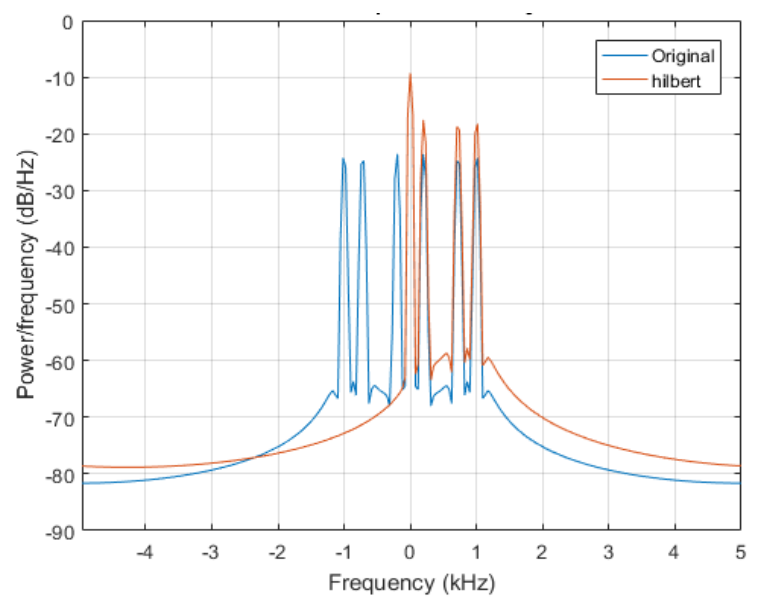

Figure 4. Power spectral density

Given $\mathrm{Q}=\circ$, since $(|\mathrm{S}(\mathrm{w})|)$ is even, its second power is also even $\left(|\mathrm{S}(\mathrm{w})|^{2}\right)$. However, w is odd, yielding that the expression $\int_{-\infty}^{+\infty} w|S(w)|^{2} d w$ is zero. Therefore, no information is available on the mean frequency, and this relation always produces zero for real signals, which is also true for all odd momentums:

$$
<w^{1}>=0: \quad \text { 1:odd } \quad \text { First }, \text { Third, Fifth, ... momentums }
$$

The analytic signal is then employed to signify the real signal. In other words, by determining $\mathrm{Z}(\mathrm{t})$, the envelope and phase characteristics are obtained with no ambiguities (determining the instantaneous frequency). It should be noted that an analytic signal is related to the Hilbert transform as follows: 


$$
Z(t)=\frac{2}{\sqrt{2 \pi}} \int_{0}^{\infty} S(w) e^{j w t} d w
$$

In the case of an analytic signal, the inverse Fourier transform is applied to the one-sided spectrum of $\mathrm{S}(\mathrm{w})$ signal, and the resulting expression is then multiplied by two so that the relation $\mathrm{Z}(\mathrm{t})=\mathrm{s}_{\mathrm{r}}+\mathrm{j} \mathrm{s}_{\mathrm{i}}$ holds a real, and no coefficients are involved.

$$
Z(t)=s(t)+\underbrace{\frac{j}{\pi} \int_{-\infty}^{+\infty} \frac{s\left(\tau_{1}\right)}{t-\tau_{1}} d \tau_{1}}_{\text {HilbertTransform }}
$$

The parameter $\mathrm{j}$ was omitted in the Hilbert transform since our objective was to find $\mathrm{Z}(\mathrm{t})=\mathrm{S}_{\mathrm{i}}+\mathrm{S}_{\mathrm{j}}$ where $\mathrm{s}_{\mathrm{i}}$ is the imaginary part obtained by the Hilbert transform. So far, the analytic signal, and then the Hilbert transform was discussed, and our objective is to show $\mathrm{Z}(\mathrm{w})$ is the same as $\mathrm{S}(\mathrm{w})$ for positive frequencies (i.e., one-sided spectrum). By starting from the $\mathrm{Z}(\mathrm{t})$ defined earlier:

$$
\begin{aligned}
& Z(t)=\frac{2}{\sqrt{2 \pi}} \int_{0}^{\infty} S\left(w_{1}\right) e^{j w_{1} t} d w_{1} \\
& Z(w)=\frac{1}{2 \pi} \int_{w_{1}=0}^{\infty} S\left(w_{1}\right) 2 \pi \delta\left(w_{1}-w\right) d w_{1}=2 S(w) \int_{w_{1}=0}^{\infty} \delta\left(w_{1}-w\right) d w_{1}
\end{aligned}
$$

The integral of unit impulse function is equal to one given that the integration interval includes the impulse. Therefore, we need to determine whether the unit impulse occurs before or after zero.

$$
Z(w)= \begin{cases}0, & w<0 \\ 2 S(w), & w \geq 0\end{cases}
$$

As noted, the real signal spectrum becomes analytic for positive frequency.

$$
<w^{n}>=\frac{1}{E} \int|Z(w)|^{2} d w
$$

In an analytic signal, in addition to the envelope, phase, and instantaneous frequency, the central momentums or moments should be determined. In the Figures 5, 6, and 7, the simulation of signal imaginary part separation is used for statistical characterization by the Hilbert transform.

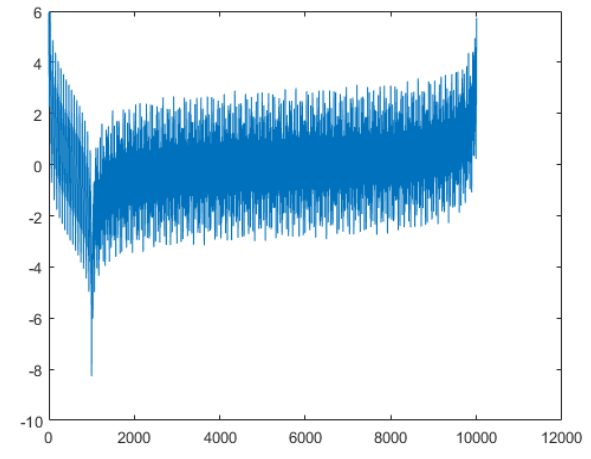

Figure 5. Hilbert transform of X signal in time domain

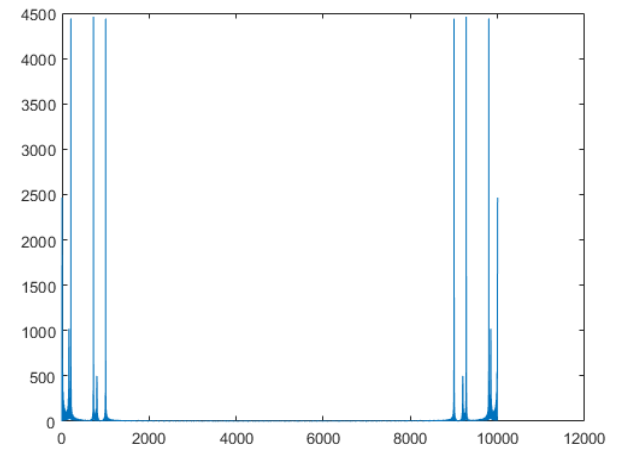

Figure 6. Fast Fourier transform of the Hilbert transform of $\mathrm{X}$ signal in frequency domain 


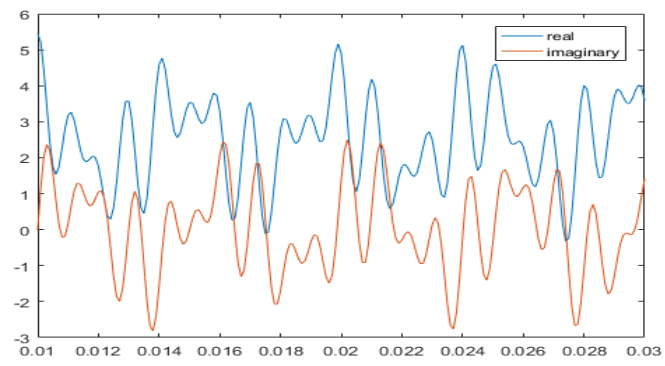

Figure 7. Hilbert function for comparison of real and imaginary signals in time domain

\section{PROPOSED METHOD FOR IDENTIFICATION OF MONO AND MULTI-COMPONENT SIGNALS}

A signal is said to be mono-component in case the distribution of energy at a given time is concentrated around a single frequency and, on the other hand, if the distribution is centered around two or more frequencies, the signal is of multi-component type. This means that our path crosses from the timefrequency domain for components analysis.

$$
\begin{aligned}
& Q=\frac{1}{E_{b}} \int s^{*}(t) \cdot \frac{1}{j}\left[\frac{d s(t)}{d t}\right] d t=\frac{1}{E_{b}} \int \varphi^{\prime}(t)|s(t)|^{2} d t \\
& =\int Y^{\prime}(t) \cdot \frac{|s(t)|^{2}}{E_{b}} d t=<\varphi^{\prime}(t)>
\end{aligned}
$$

$\mathrm{S}(\mathrm{t})$ is composed of $\mathrm{M}$ components. In a general case, we may write:

$$
\begin{gathered}
s(t)=Y(t) e^{j \varphi(t)}=\sum_{l=1}^{M} a_{l}(t) e^{j \varphi_{1}(t)} \\
\varphi^{\prime}(t)=\frac{d \varphi(t)}{d t} \neq \sum_{l=1}^{M} a_{1}(t) \varphi_{M}^{\prime}(t)
\end{gathered}
$$

\subsection{Check the reliability of the subsystem affected by the interference}

Interferences are classified into two types, namely conductive and radiated types, with the latter appearing in high-frequency telecommunication systems. Many engineers and designers may believe their system usually operates within the considered range while neglecting the fact that the system may be prone to faults undetectable by us. This can negatively affect system performance in the long run and cause the system to produce critical outputs under real-world environmental conditions. Therefore, system reliability is among the factors analysis to ensure the stability of the system. To examine system reliability, the mean time between failures (MTBF) of two or more signals is calculated, and the fault tolerability of the system in the presence of interference is determined. In other words, the ability of the system to continue its operation in the presence of interference without experiencing any hardware or software faults is examined. The reliability of the subsystem under testing is obtained depending on the complexity level, environmental

\begin{tabular}{|c|c|c|c|c|c|c|c|}
\hline Subsystem & $\begin{array}{c}\text { Spread } \\
\left(\mathrm{r}_{1}^{\prime}\right)\end{array}$ & $\begin{array}{c}\text { Technology } \\
\left(\mathrm{r}_{2}^{\prime}\right)\end{array}$ & $\begin{array}{l}\text { Operation } \\
\text { Time }\left(r_{3}^{\prime}\right) \\
\end{array}$ & $\begin{array}{l}\text { Enviromental } \\
\text { Condition }\left(r_{4}^{\prime}\right)\end{array}$ & $\begin{array}{c}\text { Overall Rate } \\
\left(\mathrm{W}_{\mathrm{k}}^{\prime}\right)\end{array}$ & $\begin{array}{c}\text { Complexity } \\
\left(\mathrm{C}_{\mathrm{k}}^{\prime}\right)\end{array}$ & $\begin{array}{c}\text { Failure Rate } \\
\left(\chi_{\mathrm{k}}\right)\end{array}$ \\
\hline $\mathrm{A}$ & 3 & 6 & 3 & 7 & 378 & 0.0993691 & 50.96967739 \\
\hline B & 6 & 3 & 9 & 4 & 648 & 0.170347 & 87.37658981 \\
\hline $\mathrm{C}$ & 5 & 5 & 4 & 5 & 500 & 0.1314406 & 67.42020819 \\
\hline D & 2 & 6 & 10 & 5 & 600 & 0.1577287 & 80.90424982 \\
\hline $\mathrm{E}$ & 1 & 4 & 9 & 3 & 108 & 0.0283912 & 14.56276497 \\
\hline $\mathrm{F}$ & 4 & 5 & 6 & 8 & 960 & 0.2523659 & 129.4467997 \\
\hline G & 2 & 3 & 5 & 7 & 210 & 0.055205 & 28.31648744 \\
\hline $\mathrm{H}$ & 1 & 8 & 5 & 10 & 400 & 0.1051525 & 53.93616655 \\
\hline Total & & & & & 3804 & 1 & 512.9329439 \\
\hline
\end{tabular}
conditions, and operating time. In Table 1 shows the Failure rate and complexity calculations in a subsystem under testing for eight subsystems.

Table 1. Failure rate and complexity calculations in a subsystem under testing 
In the table 2 shows the Reliability calculations in a subsystem under testing with used components for different quantities.

Table 2. Reliability calculations in a subsystem under testing with used components

\begin{tabular}{ccccccc}
\hline Item & QTY & $\begin{array}{c}\text { MTBF (Hrs) [From } \\
\text { the Manufacturer] }\end{array}$ & $(1 / \mathrm{Hrs})$ & Effective (1/Hrs) & T (Hrs) & R(t) (Reliability) \\
\hline RS232 Transceiver & 1 & 2290000000 & $4.36681 \mathrm{E}-10$ & $4.36681 \mathrm{E}-10$ & 100000 & 0.999956333 \\
4 bit voltage trans. & 1 & 966100000 & $1.03509 \mathrm{E}-09$ & $1.03509 \mathrm{E}-09$ & 100000 & 0.999896496 \\
3.3V regulator & 1 & 1340000000 & $7.46269 \mathrm{E}-10$ & $7.46269 \mathrm{E}-10$ & 100000 & 0.999925376 \\
Diode & 1 & 98039215 & $1.02 \mathrm{E}-08$ & $1.02 \mathrm{E}-08$ & 100000 & 0.998980520 \\
Transistors & 2 & 370370370 & $2.7 \mathrm{E}-09$ & $5.4 \mathrm{E}-09$ & 100000 & 0.999460146 \\
Resistors & 10 & 270270270 & $3.7 \mathrm{E}-09$ & $3.7 \mathrm{E}-08$ & 100000 & 0.996306837 \\
Capacitors & 15 & 2683123155 & $3.72 \mathrm{E}-10$ & $5.5905 \mathrm{E}-09$ & 100000 & 0.999441106 \\
Sum & 31 & 8017903010 & $1.91907 \mathrm{E}-08$ & $6.0485 \mathrm{E}-08$ & 100000 & 0.9939773553203 \\
\hline
\end{tabular}

\subsection{Proposed Method for Practical Simulation}

Here, is proposed a practical way for fault data analysis and input signals to the subsystem. Collection of fault data from an electronic system helps to ensure the system reliability during its design. Achieving high-efficiency reliability is impossible without collecting, analyzing, and using the acquisitive data during the testing and performance of the system. In Figures 8 and 9 show Practical identification of fault data in the unfavourable and favorable case to good and bad conditions.

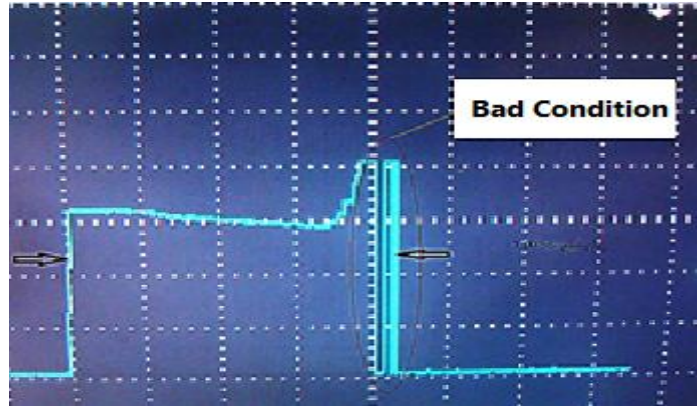

Figure 8. Practical identification of fault data in the unfavorable case

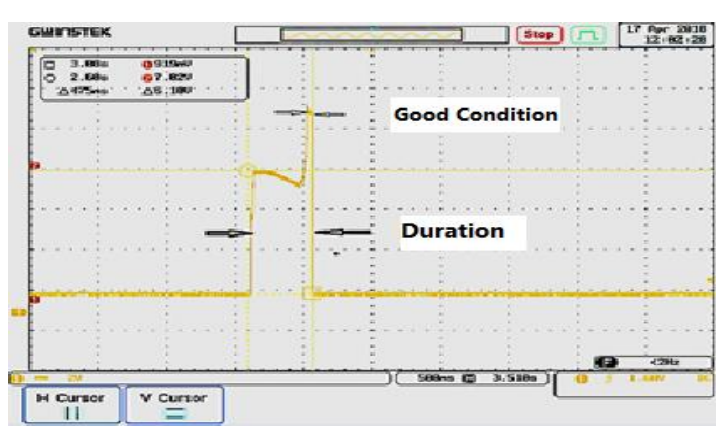

Figure 9. Practical identification of fault data in a favorable case

\subsection{Detection of the phase difference between two signals}

This method aims to detect and measure the real phase difference between the signals with different phases and amplitudes input to a subsystem under testing. Note that the detection of the phase difference between two signals can be extended to multiple signals. In this method is used from microcontroller interruption to determine zero-crossing of the signal under testing. When the signal becomes greater than zero in the positive half-cycle, the comparator circuit produces a pulse as the output. Then, by passing through a differentiator, the moment at which the signal jumps from zero to a positive level is extracted from the waveform and reported to the microcontroller, which triggers an interruption in the microcontroller, during which the considered timer is activated. When the second signal reaches the zero-crossing moment, a second interruption procedure is executed which stops the timer. The number counted by the timer is used as the criterion to determine the time difference between two signals at a specific moment. In the processing procedure, given the signal period, the phase difference is calculated in radian. For determine the phase difference between two signals with arbitrary frequencies, the signal period should be necessarily measured in the first place. In Figure 8 shows the Measuring the phase difference between two signals with identical distribution entering a subsystem. 


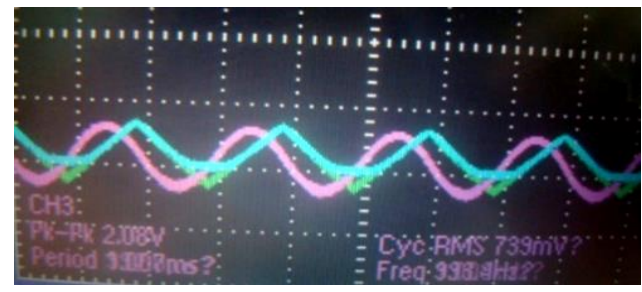

Figure 10. Measuring the phase difference between two signals with identical distribution entering a subsystem

The ability to measure the phase difference between two auto-range signals with an input amplitude of $100 \mathrm{mV}$ and a precision of 1 degree is required. To this end, the small input signal should be amplified in the first stage. The two input signals, as demonstrated, are then transformed into square waves, such that the signal edges are fully sharpened using the Schmitt Trigger unit. The prepared signals are then transmitted to the digital unit to extract the phase information. In the digital unit, the phase difference between the two signals is transformed into a square wave through XOR, and the area under the signal curve is calculated using the integrator. The resulting analog value is converted into a digital value and transferred to the microcontroller unit for processing. Ultimately, the phase difference between the two or more signals is displayed on the liquid crystal display (LCD). A phase detector circuit using XOR gate is shown in Figure 11.

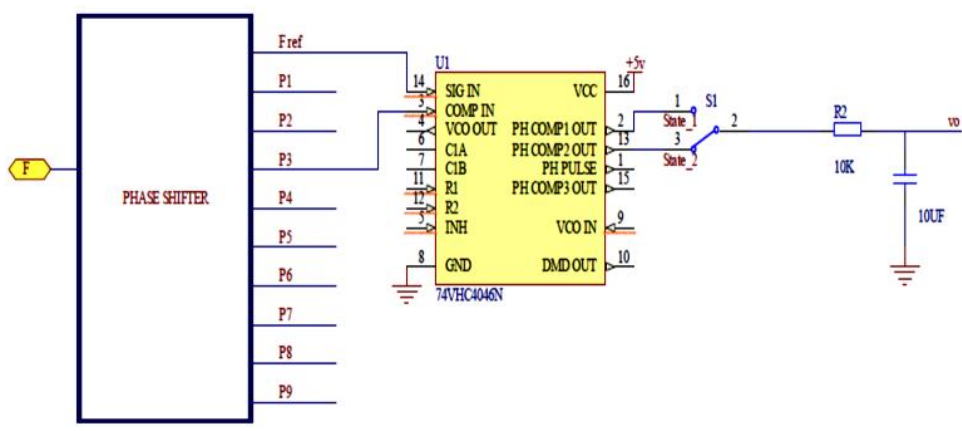

Figure 11. Phase detector using XOR

In the synchronization or phase locking mode, the phase difference between the oscillator output and the reference signal is zero or very small. In the case of the phase difference increases, the control mechanism manipulates the oscillator such that the error is minimized. In this phase control mechanism, the output signal is locked on the reference signal phase. In some of the phase lock loop (PLL), voltage controlled oscillator (VCO) is used instead of the current controlled oscillator (CCO), in which case the phase detector output is a controlled current source. However, a similar performance obtained in both cases. Proportional derivative (PD) is responsible for comparing the phases of the output and the reference signals. The PD relation is proportional to the phase difference within a specified interval. The output signal produced by the PD is composed of a dc component and an undesirable ac component that is eliminated using a usually first-degree low-pass filter. The 74HC4046 IC is of PLL type, the XOR gate of which was used in our case. In the phase shifter block, the phase difference between the two signals is generated and applied to the IC. The function of an XOR gate is very similar to a linear multiplier. In this method, the phase difference between two or more signals is transformed into a square wave using XOR, and the area under the signal curve is calculated using an integrator. Then, by converting the obtained analog value to a digital value, the microcontroller unit carries on the processing operations and calculates the phase difference. In Figure 12 shows the Calculation of phase difference using XOR gate for two signals [21]. 


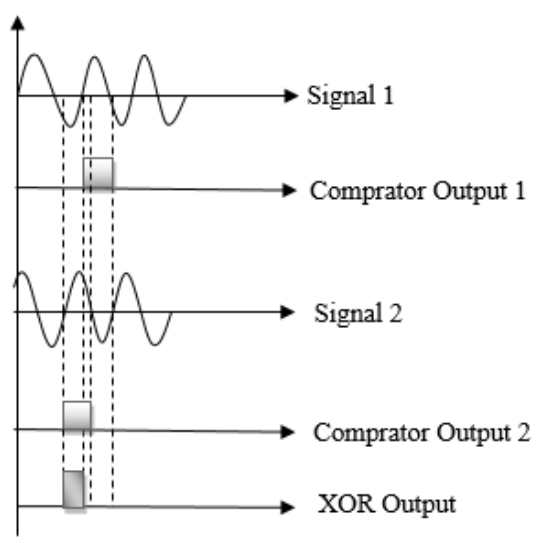

Figure 12. Calculation of phase difference using XOR gate [21]

In Figure 13 shows the block diagram of the system used for measuring the phase difference between two signals input to the subsystem.

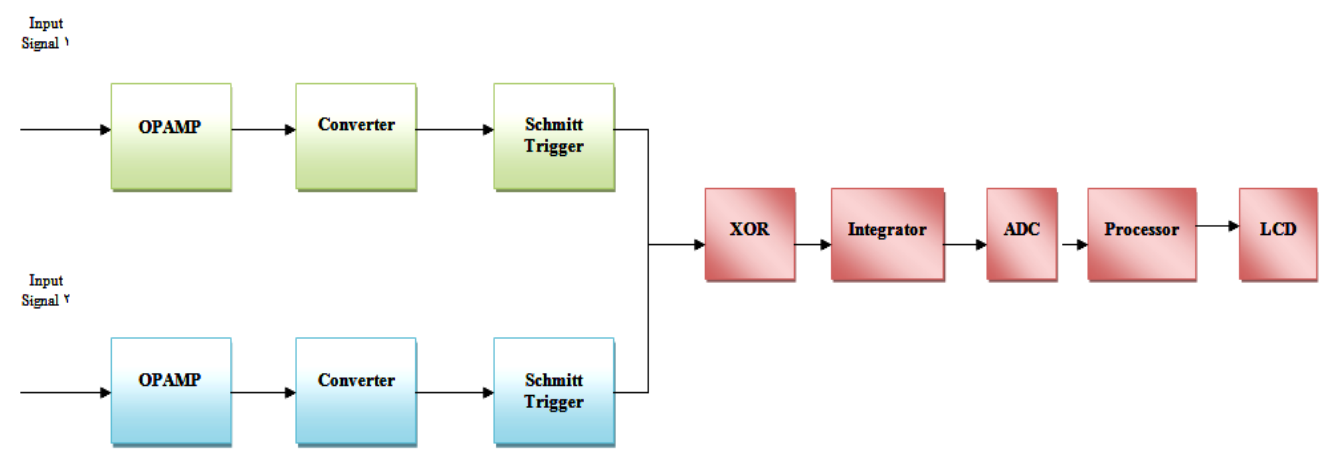

Figure 13. The overall block diagram of the system used for measuring the phase difference between two signals input to the subsystem (extendable to $\mathrm{n}$ signals)

\subsection{Proposed Algorithm}

Here, an algorithm was proposed based on the switch matrix to relate the different components and parameters. The switch matrix method actually, is used in a communication system for parameter measurement connects a device on a certain row to a signal on a particular column. There are more signals than devices. The number of rows $\mathrm{M}$ and columns $\mathrm{N}$ defines the size of the matrix as $\mathrm{M} * \mathrm{~N}$. By convention, rows are listed before columns. This method is used four different situations for example signal, supply, connection, and BNC connection field Matrices that is shown in Figure 14 and by relation of different parts, we can compute more information about parameters results. A matrix that allows every input to be connected to a separate output is described as non-blocking and can be very helpful in complex communication systems. In Figure 15 shows the switch matrix demonstration for multiple modes using four different situations for example signal, supply, connection, and BNC connection field Matrices [20]. In the software design, we use different modes for connections that every relationship between situations called Relay. sTo relate the different components and parameters is created by 35 modes that are shown in Figure 16. 


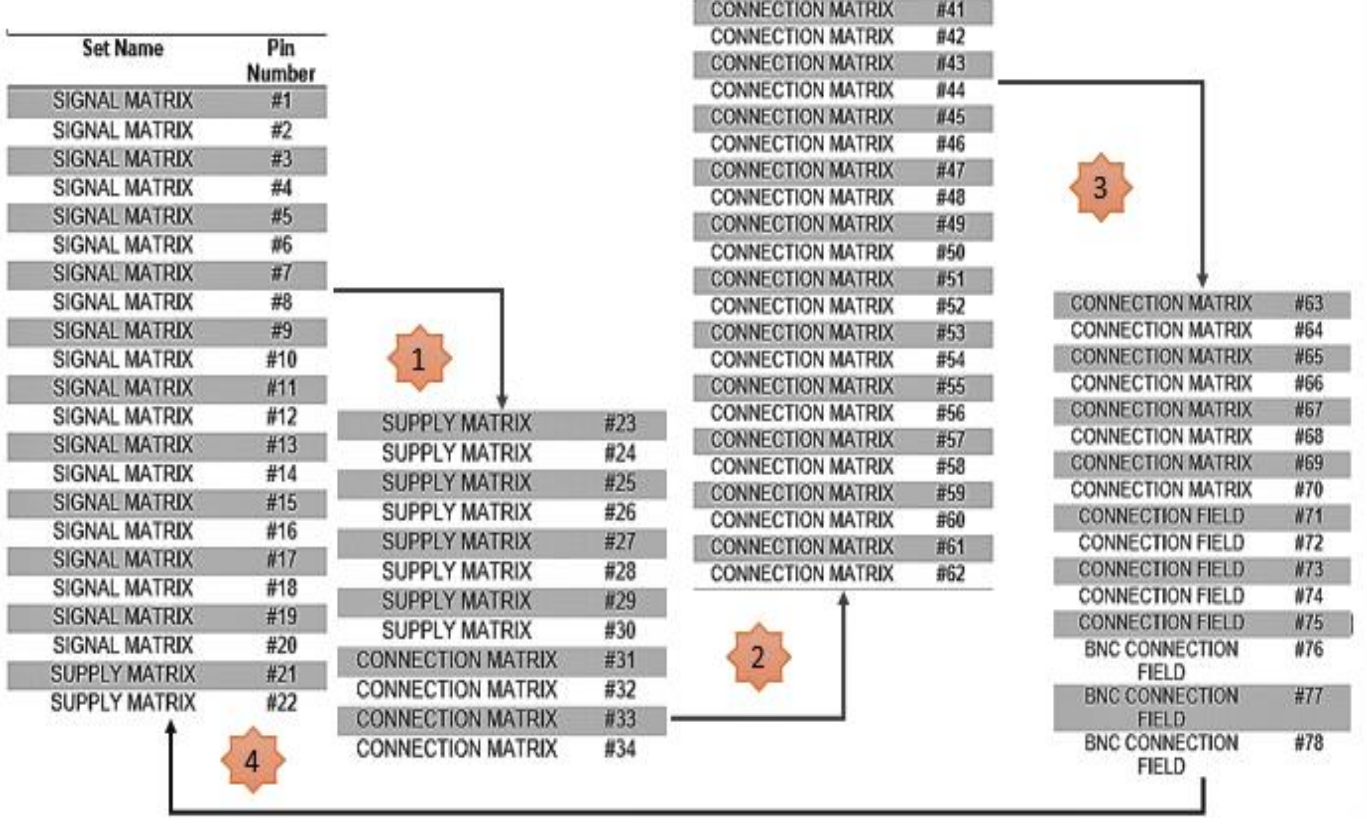

Figure 14. Switch matrix algorithm to relate the different components and parameters

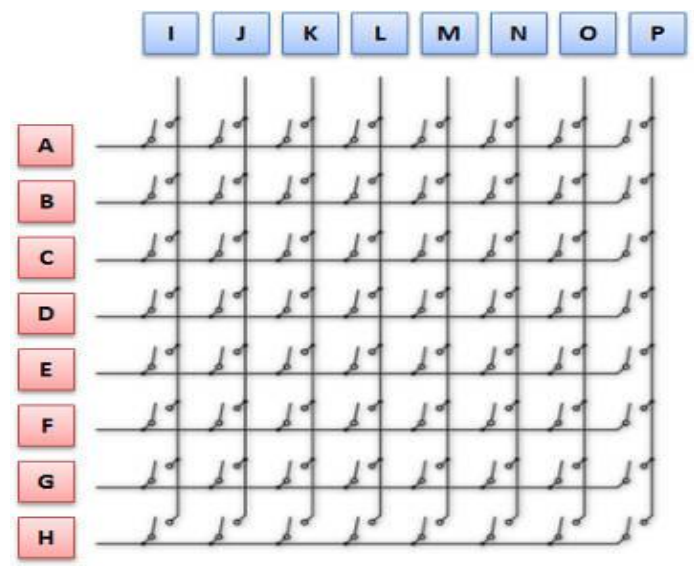

Figure 15. Switch matrix demonstration for multiple modes [20] 


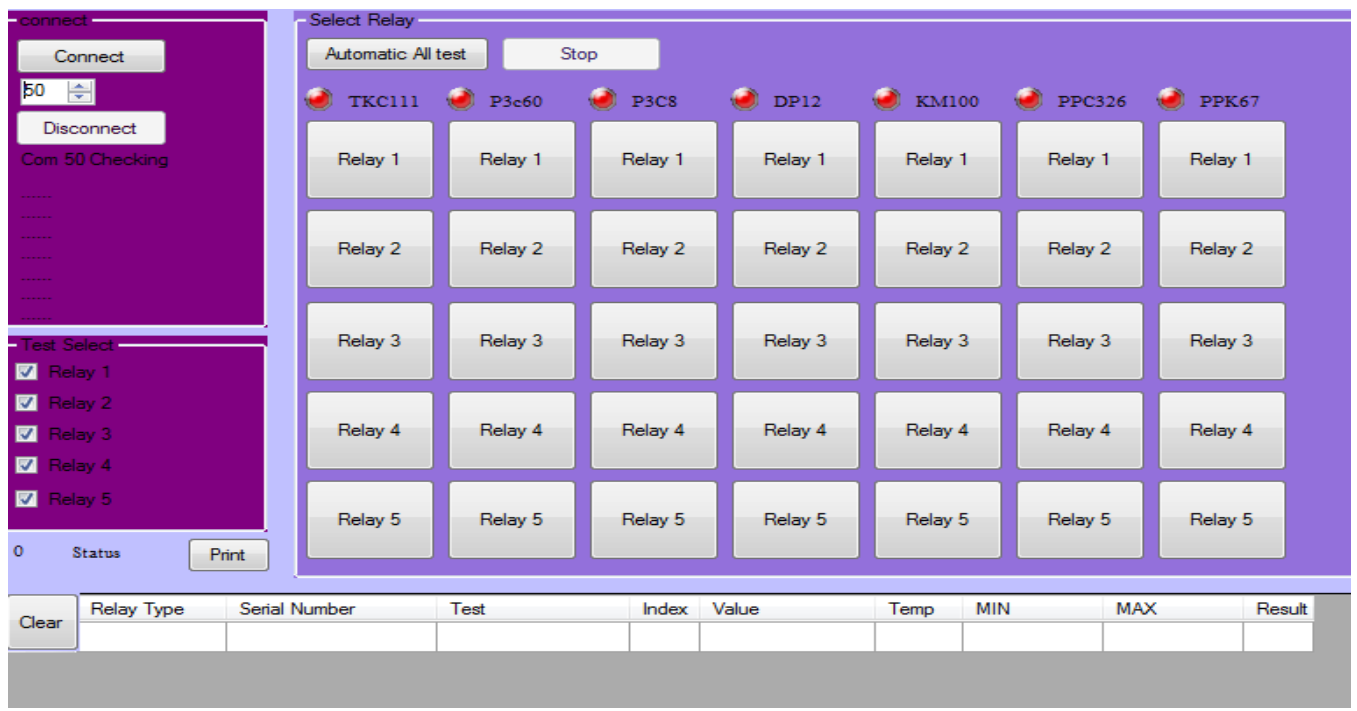

Figure 16. The software native design for performance evaluation of algorithm

\section{CONCLUSION}

In this paper, the design parameters in the modeling procedure were specified based on the system type. The signal was decomposed into various components using statistical analysis. The phase difference between the received signals was measured by analyzing a high-frequency telecommunication subsystem. The separability of the signal components and the reliability of the subsystem to assess its effectiveness in the presence of interference were also discussed. In this study, mono-component signals were extended to multicomponent ones, and the analytic signals were used to extract the statistical information of the signals in the time-frequency period. To investigate and provide a defect detection solution, the switch matrix containing all information about the defect status was used in $\mathrm{n}$ tests to analyze different scenarios and an algorithm was proposed to relate the different components and parameters. Also, a mathematical model based on the statespace equations was employed to evaluate the nonlinear modes of the system. Given that decoupling, the measurement parameters is a problem to be tackled from multiple aspects, the costs, test duration, and reliability were also taken into calculations in addition to considering all the detection methods for interference signals.

\section{REFERENCES}

[1] J. Preibisch, et al., "Corrections to An Efficient Analysis of Power/Ground Planes with Inhomogeneous Substrates Using the Contour Integral Method," IEEE Transactions on Electromagnetic Compatibility, vol. 59, pp. 1000, Jun 2017.

[2] W. A. Radasky and M. Bäckström, "Brief Historical Review and Bibliography of Intentional Electromagnetic Interference (IEMI)," XXXIth URSI General Assembly and Scientific Symposium (URSI GASS), Beijing, China, Aug 2014.

[3] Q. Chen, et al., "Amplitudes of mono-component signals and the generalized sampling functions," Signal Processing, vol. 94, pp. 255-263, Jan 2014.

[4] A. P. Albright, "A Quantitative Measure of Mono-Componentness for Time-Frequency Analysis," PhD diss., University of Tennessee, 2016.

[5] H. Li, et al., "Mono-component decomposition of signals based on blaschke basis," International Journal of Wavelets Multiresolution and Information Processing, vol. 5, pp. 941-945, Nov 2007.

[6] M. F. Sultan and A. C. Cangellaris, "System Level Approach for Automotive Electromagnetic Compatibility," IEEE Symposium on Electromagnetic Compatibility, pp. 210-220, Aug 1987.

[7] B. Moore, "Principal component analysis in linear systems: Controllability, observability, and model reduction," IEEE Transactions on Automatic Control, vol. 26, Feb 1981.

[8] ITU, "High-power electromagnetic immunity guide for telecommunication systems," ITU-T, K.81, 2015.

[9] X. Zhao, et al., "Low-Power Clock Tree Design for Pre-Bond Testing of 3-D Stacked ICs," IEEE Transactions on Computer-Aided Design of Integrated Circuits and Systems, vol. 30, pp. 732-745, May 2011.

[10] C. Schuster, "Using the Contour Integral Method for Solving Large EMC Problems on Printed Circuit Boards," Workshop on Computational Electromagnetics and Multi-physics Methods for Characterizing Complex EMC/EMI Effects (WS8) at the IEEE Symposium on Electromagnetic Compatibility, Dresden, Germany, Aug 2015. 
[11] O. M. Ramahi and B. Archambeault, "Adaptive absorbing boundary conditions in finite-difference time domain applications for emc simulations," IEEE Transactions on Automatic Control, vol. 37, pp. 580-583, Nov 1995.

[12] P. Singh, et al., "Fourier decomposition method for nonlinear and non-stationary time series analysis," Proceedings in Mathematical, Physical and Engineering Sciences, vol. 473, pp. 1-14, Mar 2017.

[13] M. Heddebaut, et al., "Method for detecting jamming signals superimposed on a radio communication, Application to the surveillance of railway environments," IEEE International Symposium on Electromagnetic Compatibility (EMC), Dresden, Germany, Aug 2015.

[14] X. Zhao and S. K. Lim, "Power and slew-aware clock network design for through-silicon-via (TSV) based 3D ICs," 15th Asia and South Pacific Design Automation Conference (ASP-DAC), Taipei, Taiwan, pp. 175-180, Jan. 2010.

[15] R. L. Chen, et al., "Analytical Model for the Rectangular Power-Ground Structure Including Radiation Loss," IEEE Transactions on Electromagnetic Compatibility, vol. 47, pp. 10-16, Feb 2005.

[16] R. F. Harrington, "Field Computation by Moment Methods," Wiley-IEEE Press, New York, 1968.

[17] C. Schuster, "Signal Integrity Engineering for High-Speed Links," Global University Lecture at the IEEE Symposium on Electromagnetic Compatibility, Dresden, Aug 2015.

[18] J. Kim, "On-Chip Measurement of Jitter Transfer and Supply Sensitivity of PLL/DLLs," IEEE Transactions on Circuits and Systems II: Express Briefs, vol. 56, pp. 449-453, Jun 2009.

[19] B. Ninness and F. Gustafsson, "A unifying construction of orthonormal bases for system identification," IEEE Transactions on Automatic Control, vol. 42, pp. 515-521, Apr 1997.

[20] S. Ramo, et al., "Fields and Waves in communication electronics," 3rd Edition, John Wiley \& Sons Inc., New York, 1994.

[21] C. Paul, "Introduction to electromagnetic compatibility,” John Wiley \& Sons Inc., New York, 1992. 